Adenokarzinom $\mathbf{1 0} 515$

Adjuvante Therapie 1-2 55

Ältere Patienten 10515

Akute myeloische Leukämie 12657

Amphotericin B 4185

Angiogeneseinhibitor 5260

Angiogeneseinhibitoren 10519

Angst 11551

Antiangiogene Therapie 12629

Antimykotische Therapie, empirische 4185

Antineoplastische Substanzen 12651

Aromataseinhibitoren 1-2 55

$\beta$-hCG 6330

Behandlungsergebnisse 12611

Behandlungsqualität $\mathbf{7 3 6 7}$

Belastung 11551

Beta-Aktin 6295

Bevacizumab 10509

Bildgebung, präoperative 3141

Bleomycin 6320

Body-Mass-Index 10 489, 11538

Bortezomib 4 193, 12651

BRCA1 1-2 14

BRCA1-Mutation 6316

Bronchialkarzinom, kleinzelliges 6311

Brustkrebs 3 134, 4 177, 5 243, 8-9 429

Brustkrebs, sporadischer 1-2 14

Bulgarische Population 11559

B-Zell-Lymphom, diffus großzelliges 3138

Capecitabin $\mathbf{1 2} 629$

Carbohydrat-Antigen 19-9 1-2 39

Celecoxib 12629

c-erb-B2 10495

Cetuximab 3 129, 10 509, 12637

Chemotherapie $\mathbf{3} 134, \mathbf{3} 138,4209$, $5228, \mathbf{5} 235, \mathbf{5} 253, \mathbf{8}-9452$

Chirurgische Therapie 5228

Cholangiokarzinom 3129

Chondrosarkom 4201

Ciclosporin 1-2 49

CLL 11564

CML 11574

CT 3141

Cyclophosphamid 8-9 452
Cystosarcoma phylloides: Differentialdiagnostik, operatives Management, Prognose 3113

Cytochrom P450 11559

Dermatitis 4209

Dihydropyrimidindehydrogenase (DPD) 6295

Docetaxe 7355

Docetaxel: wöchentliche Gabe 8-9436

Duktoskopie 5243

Duktoskopie: interventionelle, diagnostische, zukünftige technische Entwicklung 11545

EGFR-Inhibition 7355

Endometriumkarzinom 10495

Enukleoresektion 397

Epidemiologie 11538

Epidermaler Wachstumsfaktor-rezeptor 3129

Erhaltungstherapie 5265

Erlotinib (Tarceva ${ }^{\circledR}$ ) $\mathbf{1 0} 515$

Erstlinientherapie 10515

Erythropoietin 6303

Expression 6295

5-Fluorouracil 8-9 421

5-Fluorouracil, kontinuierliches intravenöses 5235

Fall-Kontroll-Studie $\mathbf{1 1} 538$

Fatigue bei Tumorkrankheit 8-9 429

FDG-PET (F-18-deoxyglucose positron emission -tomography) $\mathbf{3} 141$

Fibroadenom 3113

Fieber, ungeklärtes 4185

Fludarabin 1-2 49

Fluoropyrimidine 6295

Fortgeschrittenes Nierenzellkarzinom 8-9 450

Galaktografie 5243

GAPDH 6295

Gefitinib 7355

Gemcitabin 1-2 39, 5253

Gemtuzumab Ozogamicin 12657

Genexpressionssignatur 8-9 421

Gewebedifferenzierung $\mathbf{7 3 8 8}$
Gezielte Therapie 5265

GIST 11568

GIST, c-kit-negativ 12645

Graft-versus-Myelom-Effekt 4193

Gute Klinische Praxis 1-2 21

\section{HART 10479}

Hauspflege 3105

HEA125 6311

Hirnmetastasen 7361

Hodgkin-Lymphom 5265

Hormonrezeptoren 1-2 55

Hormontherapie 5249

Hyperbilirubinämie $\mathbf{1 0} 509$

Hyperfraktionierte akzellerierte Radiotherapie $\mathbf{1 0} 479$

Hyperthyreose 6330

Hyponatriämie $\mathbf{1 2} 651$

Imatinib 7381

Imatinib-Resistenz 11574

Immunhistochemie $\mathbf{1 0} 495$

Immunzytologie $\mathbf{6} 311$

Irinotecan 4 169, 12637

Itraconazol 4185

Junge Patientinnen 10502

Kardiotoxisch 3134

Keimzelltumoren $\mathbf{6} 330$

Kinderonkologie 1-2 21

Klinische Prüfung, nicht kommerzielle 1-2 21

Knochenmarksveränderungen $\mathbf{6} 303$

Kolonkarzinom 12637

Kolorektales Karzinom 4 169, 7 385, $\mathbf{1 1} 559$

Kompetenznetz 5265

Kopf-/Hals-Tumoren, lokal fortgeschritten $\mathbf{1 0} 479$

Körperliches Training 8-9 429

Kosten-Nutzen-Analyse 12621

Lebenserwartung 8-9 414

Lebensqualität $\mathbf{4} 177$

Lebermetastasen $\mathbf{1 0}$ 509, 12637

Leiomyosarkom 12645

Lendenwirbelsäule 6303

\title{
KARGER
}

(C) 2007 S. Karger GmbH, Freiburg 
Leukenzephalopathie-Syndrom, reversibles posteriores $\mathbf{3} 138$

Leukoenzephalopathie 1-2 49

Liposomales Amphotericin B 12621

Lungenkarzinom, nichtkleinzelliges 10515

Lungenkrebs 7375

Lymphom der Brust, primäres 3134

Magen-Darm-Krebs 8-9 421

Magenkarzinom 1-2 29

Magenkarzinom, fortgeschrittenes 5235

Magenkrebs 11568

Maligne Lymphome 5265

Maligner Phyllodestumor 1-2 45

Mamillensekretion 5243

Mammakarzinom 8-9 443, 8-9 455

Mammakarzinom, «basal-like» 6316

Mammakarzinom, metastasiertes 8-9 436

Mammakarzinom, primäres 1-2 55

Management 11574

Mantelzell-Lymphom 12651

Melanom des Ösophagus, asymptomatisches malignes 4206

Meningeosis 11564

Meningeosis carcinomatosa 8-9 450

Metachron 11568

Metastasen der Bauchlymphknoten 7375

Metastasen: Gesicht 6324

Metastasiert 5260

Methotrexat 5228

Metronomische Therapie 12629

Milchgangsendoskopie $\mathbf{1 1} 545$

Milchgangsuntersuchung, diagnostische 11545

Milz 5256

Mitomycin C 1-2 29, 5235

MOC-1 6311

Molekular-pathologische

Charakteristika 10502

MRI 3141

MRT 6303

Mutationen 11574

Nachsorge 8-9 443

Nebenwirkungen 10519

Nebenwirkungsprofil 8-9 436

Nephron-erhaltende Chirurgie 397

Neuroborreliose 11564

Neurotoxische $\mathbf{3} 134$

Neutropenie 4185, 12621
Nierenzellkarzinom 3 97, 5260

Non-Hodgkin-Lymphom $\mathbf{7} 385$

Obesität 11538

Onkologen 7367

Onkologische Rehabilitation 11551

Ösophagektomie 4206

Osteosarkom 5228

Ovarialkarzinom 12611

Oxaliplatin 1-2 29

p16 1-2 14

Palliativversorgung 3105

Pankreaskarzinom 1-2 39

Papillom 5243

Paravasat 8-9 452

PDGFRA-Färbung 12645

Penismetastase 5249

PET-CT 7 375, 12645

Pharmaforschung $\mathbf{7 3 8 8}$

Phase-II-Studie 1-2 21, 5 235, 12637

Plasmazellleukämie 4193

Plattenepithelkarzinom der Mamma 6316

Plattenform 3121

Plattenkomplikationen 3121

Pneumonitis 6320

Polymorphismen 11559

Positronen-Emissions-Tomographie 6320

Positronenemissionstomographie 5265

Präoperative Chemoradiotherapie 6324

Prognose 5 260, 10502

Prognosefaktoren 5228

Prognose-Indikator 10495

Prognose-Score 8-9 414

Prognostischer Faktor 1-2 39

Promotor 8-9 421

Prophylaxe $\mathbf{1 2} 621$

Prospektive, randomisierte Studie 4177

Prostatakarzinom 7 355, 10 489, 11568

Prostatakarzinom, inzidentell 5249

Prostatakrebs 11551

Prostatektomie, radikale 10489

Radiotherapie 3 134, 5 249, 7361

RCC 10519

Recall-Reaktionen 4209

Rehabilitation 8-9 429

Rekonstruktionsplatte $\mathbf{3} 121$

Rekonstruktive Chirurgie 7388

Rektumkarzinom 3 141, 6324
Rheinland-Pfalz 12611

Rituximab 3138

RT-PCR 6295

14-3-3o 1-2 14

Sarkom der follikulären dendritischen Retikulumzellen 7381

Schraubenlockerung 3121

Schrittmacher mit Defibrillatorfunktion 7378

Seminom 6320

SIADH 8-9 455

Siganlintensitätsveränderungen $\mathbf{6} 303$

Sorafenib 10519

Sorafenib 8-9 450

Speicheldrüse 6324

Spindelzelltumoren $\mathbf{7 3 8 1}$

Staging 10489

Staging: lokoregional, Fern- 3141

Stammzelltransplantation, allogene 4193

Stationäre Rehabilitation 4177

Sterbeorte von Krebspatienten $\mathbf{3} 105$

Strahlentherapie 4 209, 7378

Sunitinib 10519

Syndrom der inadäquaten Sekretion des antidiuretischen Hormons (SIADH) 12651

Temozolomid 7361

Testsysteme $\mathbf{7 3 8 8}$

Thymidylatsynthase (TS) 6295

Tissue Engineering 7388

Transkriptionsfaktor 8-9 421

Trofosfamid $\mathbf{4} 201$

Tumorinzidenz $\mathbf{1 1} 538$

Tumormarker 8-9 443

Tumorsuppressorgene, Hypermethylierung 1-2 14

Überleben 5260

Überlebenszeit 8-9 443

Uferzellangiom, multiples 5256

Ultraschall, endorektaler 3141

Umfrage 7367

Unterkieferteilresektion $\mathbf{3} 121$

Vinorelbin 8-9 455

Vorhofflimmern 5253

Wirbelsäulenmetastasen 8-9 414

Zervixkarzinom 10502

ZNS 11564

Zweitlinientherapie $\mathbf{4} 169$ 